\title{
Carbapenemase-producing Pseudomonas aeruginosa from central Greece: molecular epidemiology and genetic analysis of class I integrons
}

Apostolos Liakopoulos ${ }^{1,2}$, Angeliki Mavroidi ${ }^{1}$, Efstathios A Katsifas' ${ }^{2}$, Alexandros Theodosiou ${ }^{3}$, Amalia D Karagouni ${ }^{2}$, Vivi Miriagou ${ }^{4}$ and Efthymia Petinaki, ${ }^{1,}$

\begin{abstract}
Background: Multidrug-resistant Pseudomonas aeruginosa is a serious challenge for antimicrobial therapy of nosocomial infections, as it possesses several mechanisms of antimicrobial resistance. In Central Greece, a sudden increase of infections caused by carbapenem-resistant P. aeruginosa was observed during 2011, indicating the need for further analysis.

Methods: Five-hundred and sixty-eight $P$. aeruginosa isolates were collected consecutively during an 8-month period in 2011 from inpatients treated in three hospitals in the Thessaly region (1,000,000 habitants) of Greece. Carbapenemresistant $P$. aeruginosa $(n=284)$ were characterized by antimicrobial susceptibility testing and $\beta$-lactamase content, and the genetic relatedness of carbapenemase-producing isolates was assessed by BOX-PCR, multilocus sequence typing, and eBURST analysis. Mapping of the class I integrons of Verona integron-encoded metallo- $\beta$-lactamase (VIM)-carrying isolates was also performed, and clinical data of the VIM producers were reviewed.

Results: Eighty (14.1\%) out of the 568 P. aeruginosa isolates recovered from clinical specimens were VIM producers. Multilocus sequence typing revealed high prevalence of the international clones ST111 and ST235 among blaVIM-2and blaVIM-4-positive isolates, respectively. blaVIM-17 was identified in an isolate of a novel sequence type (ST1457). bla $a_{\text {II }}$ gene cassettes were carried by five distinct class I integrons, including two novel ones.

Conclusions: Since the first report of VIM-producing P. aeruginosa in 2000, this microorganism still remains among the most prevalent multidrug resistant pathogens in Greece. The spread of VIM-producers belonging to the most common international clones (ST111 and ST235), the spread of integrons of divergent structures, and the emergence of novel integrons underscore their ongoing evolution.
\end{abstract}

Keywords: Pseudomonas aeruginosa, MDR, Carbapenems, VIM, Integrons, MLST

\section{Background}

P. aeruginosa is a leading cause of nosocomial infections, especially in immunocompromised patients. The high prevalence of multidrug-resistant (MDR) $P$. aeruginosa is a serious challenge for antimicrobial therapy. MDR $P$. aeruginosa possesses several mechanisms of antimicrobial resistance; over-expression of the intrinsic AmpC-type cephalosporinase, which confers resistance

\footnotetext{
* Correspondence: petinaki@med.uth.gr

'Department of Microbiology, University Hospital of Larissa, Larissa, Greece ${ }^{5}$ Department of Microbiology, Medical School, University of Thessaly, Biopolis, Larissa, Greece

Full list of author information is available at the end of the article
}

to ceftazidime, inactivation or down-regulation of the OprD porin, conferring resistance to carbapenems, and upregulation of the MexAB-OprM or other efflux pumps of the resistance-nodulation-cell division family, which can also confer resistance to carbapenems, as well as to quinolones, and in some cases, to aminoglycosides [1].

Acquired resistance to $\beta$-lactams is often reported in $P$. aeruginosa as a result of the acquisition of extended spectrum $\beta$-lactamases (ESBLs), such as the PER-1, VEB-1, TEM, and SHV type ESBLs [2]. Acquired resistance to carbapenems can also be mediated by the production of either specific carbapenem-hydrolyzing $\beta$-lactamases

\section{Ciomed Central}


(carbapenemases), such as the metallo- $\beta$-lactamases (MBLs), including the Verona integron-encoded $\beta$ lactamase (VIM) and imipenemase (IMP), or by Klebsiella pneumoniae carbapenemase (KPC) enzymes and GES/ IBC-like enzymes [3-5]. MBLs hydrolyze almost all clinically-available $\beta$-lactams except monobactams, and the respective bla genes are often carried on transferable structures, known as integrons [4]. Nosocomial outbreaks caused by MBL-producing $P$. aeruginosa have been reported in several countries worldwide [6-10]. Implementation of multilocus sequence typing (MLST) has facilitated the elucidation of the global epidemiology and evolution of these multidrug-resistant pathogens [11-14].

In the prefecture of Thessaly (Central Greece), the rate of isolation of carbapenem-resistant $P$. aeruginosa was around 33\% during the period 2007-2010, but a sudden increase to 50\% was observed in 2011 (E. Petinaki, unpublished data). For this reason, the molecular epidemiology of carbapenemase-producing $P$. aeruginosa isolated from Thessaly was investigated. The genetic context of the bla $_{\mathrm{VIM}}$ genes was also studied.

\section{Methods}

Bacterial isolates and antimicrobial susceptibility testing

Five-hundred and sixty-eight $P$. aeruginosa isolates were collected from March to October 2011 from the same number of inpatients treated at three hospitals in Thessaly, Greece. Three hundred and fifty isolates were recovered from the University Hospital of Larissa (UHL), a 600-bed tertiary care hospital, while the remaining isolates were collected from the general hospitals of Volos and Trikala. Species identification and antimicrobial susceptibility testing were performed using the Vitek-2 automated system (BioMerieux Inc., Marcy l' Etoile, France), according to the interpretive criteria of the Clinical and Laboratory Standards Institute [15]. MDR P. aeruginosa are generally defined as $P$. aeruginosa resistant to imipenem, ciprofloxacin, and amikacin (minimum inhibitory concentrations (MICs) of $\geq 16 \mathrm{mg} / \mathrm{L}, \geq 4 \mathrm{mg} / \mathrm{L}$, and $\geq 64 \mathrm{mg} / \mathrm{L}$, respectively). ESBL production was tested using the double disk synergy test. Briefly, disks of ceftazidime, cefotaxime, aztreonam, and cefepime (30 $\mu \mathrm{g}$ each) were placed at a distance of $20 \mathrm{~mm}$ (center to center) from a disk containing amoxicillin (AMC, $20 \mu \mathrm{g}$ ) and clavulanic acid (CLA, $10 \mu \mathrm{g}$ ) on Mueller Hinton agar plates [2]. Phenotypic screening for carbapenemase production was performed by the modified Hodge-test with a meropenem disk, and by the imipenem-EDTA double-disk synergy test on Mueller Hinton agar plates [5].

\section{Detection of $\beta$-lactamase genes}

DNA was extracted from the carbapenem-resistant $P$. aeruginosa using a Quick-gDNA MiniPrep kit (Zymo Research, Murphy, USA). bla genes were detected by polymerase chain reaction (PCR), using specific primers for genes encoding MBLs (bla $a_{\mathrm{VIM}}, b l a_{\mathrm{IMB}} b l a_{\mathrm{GIM}}$, bla $\left.a_{\mathrm{NDM}}, b l a_{\mathrm{SPM}}, b l a_{\mathrm{SIM}}\right), \mathrm{KPC}\left(b l a_{\mathrm{KPC}}\right)$, ESBLs $\left(b l a_{\mathrm{PER}}\right.$, $\left.b l a_{\mathrm{VEB}}, b l a_{\mathrm{SHV}}, b l a_{\mathrm{TEM}}, b l a_{\mathrm{CTX}-\mathrm{M}}, b l a_{\mathrm{GES}}\right)$, and OXA type $\beta$-lactamases (bla $\left.a_{\text {OXA-40, }} b l a_{\text {OXA-48, }}, b l a_{\text {OXA-50 }}\right)$, as described previously $[2,4,16]$. The PCR products obtained from the VIM-positive isolates $(n=80)$ were sequenced on both DNA strands using an ABI3730 DNA sequencer (Applied Biosystems, Warrington, United Kingdom). Nucleotide and deduced protein sequences were identified by comparing the sequences with the database developed by Jacoby and Bush (http://www.lahey.org/Studies).

\section{Molecular typing of isolates}

Molecular typing was performed by BOX-PCR, as described previously [17] for carbapenemase-producing $P$. aeruginosa. MLST was performed for 55 representative of the aforementioned isolates, including all isolates of the major BOX-patterns and one isolate from each of the remaining BOX-patterns, as described previously [14]. Sequences were obtained from both DNA strands, and allelic profiles were determined and assigned to sequence types (STs). Novel alleles and STs were submitted to the P. aeruginosa MLST website (http://pubmlst.org/ paeruginosa/). Non-overlapping groups of related STs were identified using eBURST, with the default setting for the definition of groups (http://eburst.mlst.net) [18].

\section{PCR mapping of class I integrons}

Combinations of specific primers for the conserved segments (5' CS and 3' CS) of class I integrons together with the $b l a_{\mathrm{VIM}}$ primers were used for PCR mapping of class I integrons, as described previously [19]. Both DNA strands of the PCR products were sequenced using additional primers specific for the bla $a_{\mathrm{PSE}-1}, b l a_{\mathrm{OXA}-10}$, arr-7, aacA4, aacA7, smr-2, and $d f r b$ genes [19,20], using an ABI3730 DNA sequencer (Applied Biosystems).

\section{Collection of clinical data}

The medical records of patients infected with or colonized by carbapenemase-producing $P$. aeruginosa were reviewed retrospectively. Prior to obtaining the clinical information for the patients, approval was received from the Ethics Committee of the UHL, which is represented by the Infection Control Committee (permission number 1234). The patients were assumed to have acquired MDR P. aeruginosa prior to hospitalization when the microbe was isolated within $48 \mathrm{~h}$ of admission, while colonization with MDR $P$. aeruginosa was defined by the absence of relevant symptoms. Clinical information included age, duration of hospitalization, previous hospitalization history (history of transfer from another hospital, hospitalized departments, and wards), underlying diseases, clinical outcome, medical care exposures, and antimicrobial therapy. 


\section{Results and discussion}

Of the 568 P. aeruginosa isolates, 284 (50\%) were identified as resistant to carbapenems (MICs for imipenem $\geq 16 \mathrm{mg} / \mathrm{L}$ ). Of the 284 carbapenem-resistant isolates, 80 (28\%) isolates were positive for carbapenemase production by phenotypic screening and were positive for bla $a_{\mathrm{VIM}}$ by PCR. The remaining 204 carbapenem-resistant $P$. aeruginosa isolates were negative for carbapenemase production by phenotypic screening and negative for all ESBL and carbapenemase genes tested. Thus, the carbapenem resistance phenotype of the latter isolates may be attributed to other resistance mechanisms, such as porin loss, increased efflux, and AmpC overexpression.

All VIM producers exhibited MDR phenotypes. Apart from being resistant to imipenem and meropenem (MICs $\geq 16 \mathrm{mg} / \mathrm{L}$ ), all VIM producers showed resistance to ticarcillin/clavulanate (MICs $\geq 128 \mathrm{mg} / \mathrm{L}$ ) and piperacillin/tazobactam (MICs $\geq 128 \mathrm{mg} / \mathrm{L}$ ), and intermediateresistance or resistance to ceftazidime (MICs of 16 to $\geq 64 \mathrm{mg} / \mathrm{L}$ ). In addition to being resistant to $\beta$-lactams, these isolates were also resistant to ciprofloxacin (MICs $\geq 4 \mathrm{mg} / \mathrm{L}$ ), and excluding two isolates, to amikacin (MICs $\geq 64 \mathrm{mg} / \mathrm{L}$ ), gentamicin (MICs $\geq 16 \mathrm{mg} / \mathrm{L}$ ), tobramycin (MICs $\geq 16 \mathrm{mg} / \mathrm{L}$ ), and netilmicin (MICs $\geq 32 \mathrm{mg} / \mathrm{L}$ ). All VIM producers were susceptible to colistin (MICs $\leq 2 \mathrm{mg} / \mathrm{L})$. The VIM producers showed higher $\mathrm{MIC}_{50}$ values for caftazidime (MICs $\geq 64 \mathrm{mg} / \mathrm{L}$ ) and tobramycin $(\geq 16 \mathrm{mg} / \mathrm{L})$ compared with the non-VIM producing carbapenem-resistant $P$. aeruginosa (MICs of $4 \mathrm{mg} / \mathrm{L}$ and $2 \mathrm{mg} / \mathrm{L}$, respectively). No other differences were observed for the MIC range, $\mathrm{MIC}_{50}$, and $\mathrm{MIC}_{90}$ values of the remaining antimicrobial agents tested.

Of the 80 VIM producers, 48 encoded VIM-2, 31 had VIM-4, and one isolate contained VIM-17 enzymes. The monthly distribution of VIM-2 and VIM-4 producers is shown in Figure 1. An increase in the recovery rates of VIM-2 producers was observed from March to August 2011, whereas VIM-4 producers were recovered at higher frequencies in May and September 2011. The VIM producers were recovered from various wards of the hospitals, but mainly from the internal medicine (26\%) and intensive care units (ICUs) (13\%). Most of the VIM producers were isolated from urine (20.6\%), but they were also recovered from sputum, blood, bronchial secretions (13\% each), and other clinical specimens.

According to the clinical records, 52 of the 80 VIMpositive patients were infected, and 28 of 80 were colonized. A retrospective review of the clinical records of the patients infected with or colonized by VIM-producing $P$. aeruginosa was performed. All patients were diagnosed with an underlying disease at admission (such as solid tumors, diabetes, or cerebrovascular accidents), and 50\% had a high fever. All patients had a previous hospitalization history (75\% had been transferred from another hospital) and had received therapy with antimicrobial agents of multiple classes (e.g. penicillin-inhibitor combinations, second and third-generation cephalosporins, carbapenems, fluoroquinolones, glycopeptides, and cyclic lipopeptides). All but three cases of patients infected or colonized by VIMproducing $P$. aeruginosa were $>65$ years of age. The majority of infected patients were treated with a combination of colistin and aminoglycosides.

Two major BOX-PCR patterns were identified: P1 amongst VIM-2 and P2 amongst VIM-4 producers, which consisted of 21 (26\%) and 16 (20\%) out of the 80 VIM producers, respectively (Table 1 ). The remaining VIM producers were distributed into 18 different BOX-patterns, each comprised of one to five isolates. Amongst the 55 representative VIM-producers (all isolates of the major BOX-patterns and one isolate from each of the remaining BOX-patterns), we identified eight different STs. The international clones ST111 (clonal complex CC111) and ST235 (CC235) were the most prevalent, comprising 26 and 21 of the 55 VIM producers genotyped, respectively (Table 1). Both STs have been reported previously among VIM producers recovered from the university hospital of Patras, South Greece [21], and from other European countries, such as Sweden, Germany, France, Italy, Spain, and Bulgaria [12,13,21-26].

Five different class I integrons were identified: three structures coding for VIM-2 (aacA29a $\left|b_{l} a_{\mathrm{VIM}-2}\right| a a c A 29 b$, bla $a_{\mathrm{VIM}-2}|a a c A 7| d f r B$, and $\left.b l a_{\mathrm{OXA}-10}|a a c A 4| b l a_{\mathrm{VIM}-2} \mid s m r-2\right)$, one for VIM-4 (bla $\left.a_{\mathrm{VIM}-4}|a r r-7| a a c A 4 \mid b l a_{\mathrm{PSE}-1}\right)$, and one for VIM-17 (aacA29a|bla $\left.a_{\mathrm{VIM}-17} \mid a a c A 29 b\right)$ (Table 1, Figure 2). Three of the five class I integrons found here have been described previously in $P$. aeruginosa $[19,20]$, whereas in the remaining two the $b l a_{\mathrm{VIM}-2}$ gene was found as part of novel gene cassette arrays [GenBank: KC527014 and KC527015].

In the current study, the aacA29a|bla $a_{\mathrm{VIM}-2} \mid a a c A 29 b$ gene cassette array (In59-like) has been identified in 45 VIM-2 producers belonging to STs 111, 244, 253, 277, and 773. The majority of isolates assigned to ST111 were recovered from ICUs (15 out of 26 ST111 isolates; 58\%), and they were isolated mainly from bronchial secretions and blood. These results were in concordance with other studies, where, respiratory care procedures among intubated patients in the ICU have been reported to account for the spread of MDR $P$. aeruginosa, and for the high mortality rates $(40-60 \%)$ in bacterial nosocomial pneumonia and ventilator-associated pneumonia [27]. However, ST111 isolates were also found in other wards of our institutions; thus clonal expansion of VIM-2producing ST111 isolates was documented. In addition, STs 244, 253, 277, and 773 carrying the In59-like cassette array were recovered from our internal medicine units. It is known that VIM-2 producers carrying the In59-like integron have previously been reported in Greece [20, GenBank: EU118149]. Single nucleotide 

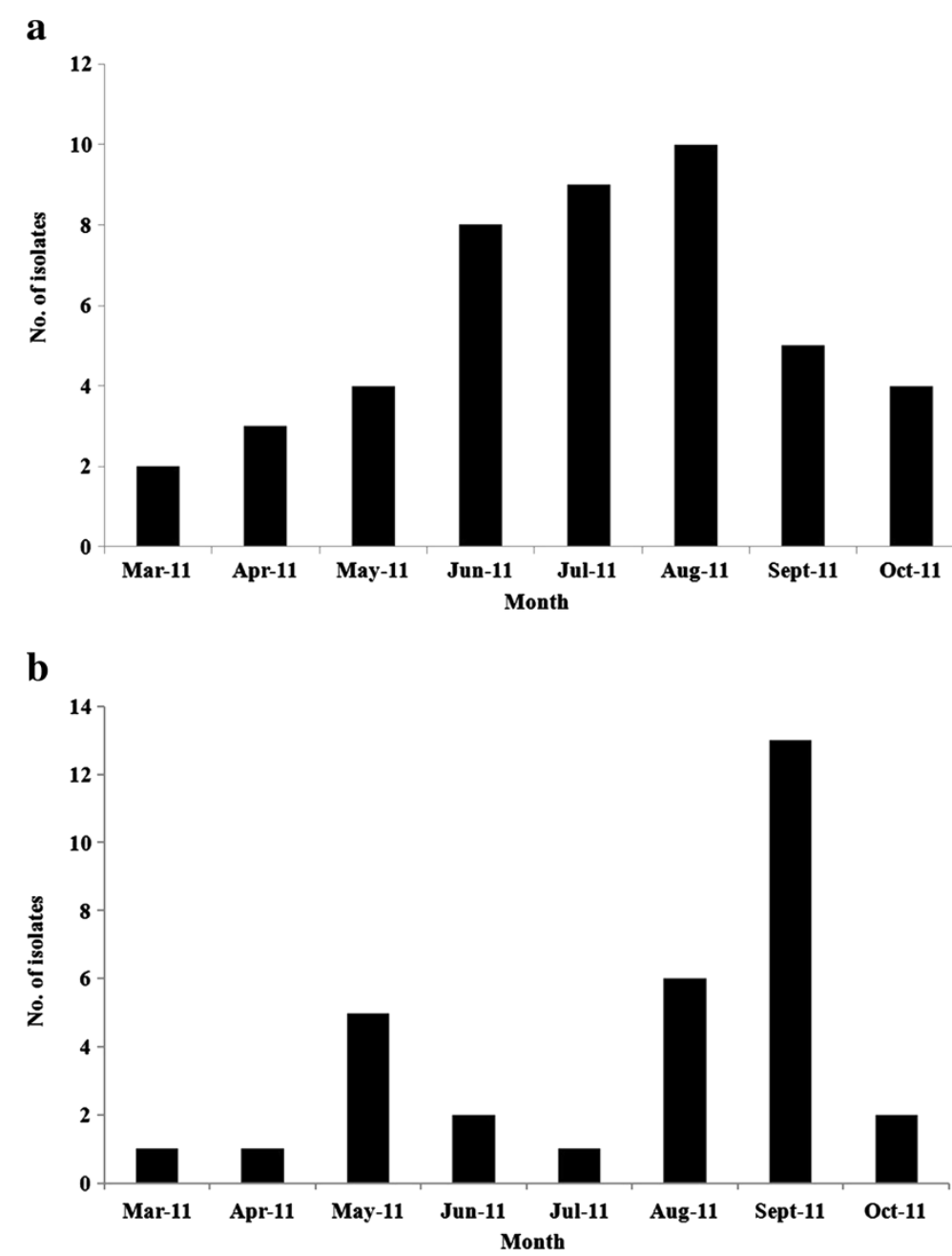

Figure 1 Monthly distribution of a) VIM-2 and b) VIM-4 P. aeruginosa from March to October 2011.

variants in the 59-base element of $a a c A 29 b$ among VIMproducers have been described in Sweden, with one isolate originating from Greece [19, GenBank: AF263519]. The presence of the In59-like cassette in different strains/genetic backgrounds underscores the recruitment potential of this integron, and may indicate its association with a mobile structure.

The $b l a_{\mathrm{VIM}-4}|a r r-7| a a c A 4 \mid b l a_{\mathrm{PSE}-1}$ gene cassette array has been described in Scandinavia, where it was identified in two MBL-producers that were imported from Greece and Cyprus, one of which was also ST235 [20, GenBank: FN397623]. In the current study, all VIM-4 producers $(\mathrm{n}=31)$ belonged to a single ST (ST235), and were isolated mainly from urine specimens (15 isolates, $50 \%$ ) from the internal medicine (12 isolates) and urology units (ten isolates). However, VIM-4 producers were also isolated from other wards of the participant hospitals, suggesting clonal expansion of a single strain. From March to August 2011, VIM-4 producers of ST235 were isolated mainly in the internal medicine unit of UHL, whereas spread of these isolates in the urology unit was documented in September 2011.

The two novel VIM-2 encoding integrons $\left(b l a_{\mathrm{VIM}-2} \mid\right.$ $a a c A 7 \mid d f r B$ and $\left.b l a_{\mathrm{OXA}-10}|a a c A 4| b l a_{\mathrm{VIM}-2} \mid s m r-2\right)$ were found in isolates of STs 235 and 395 (Table 1). The blaVIM-17 (a variant of $b l a_{\mathrm{VIM}-2}$ ) gene was found in a single isolate of a novel ST (ST1457), a single locus variant of ST235. bla $a_{\mathrm{VIM}-17}$ was carried by an In59-like structure that has also been reported in $P$. aeruginosa from Greece [20, GenBank: EU118148].

\section{Conclusions}

Multidrug-resistant VIM producers comprised $14.1 \%$ of the total $P$. aeruginosa recovered in Thessaly during 2011. Since the first report of VIM-producing $P$. aeruginosa in 2000 [28,29], this microorganism remains among the most prevalent MDR pathogens in Greece. VIM-1, VIM-2, VIM-4, and VIM-17-producing $P$. aeruginosa have been reported previously in Greece [21,28-30]. In the present study, the association between the genetic 
Table 1 Gene cassettes, STs/CCs and BOX-patterns of the VIM-producing $P$. aeruginosa isolates

\begin{tabular}{|c|c|c|c|c|}
\hline Gene cassettes $^{\mathrm{a}}$ & GenBank; reference & No. of isolates & ST/CC & BOX pattern (no. of isolates) \\
\hline \multirow{11}{*}{$a a c A 29 a\left|b l a_{\mathrm{VIM}-2}\right| a a c A 29 b$} & \multirow{11}{*}{ EU118149; [20] } & \multirow{11}{*}{45} & $111 /$ CC111 & P1 (21) \\
\hline & & & $111 /$ CC111 & P5 (3) \\
\hline & & & $111 /$ CC111 & P9 (4) \\
\hline & & & $111 /$ CC111 & P11 (2) \\
\hline & & & $111 /$ CC111 & P14 (1) \\
\hline & & & $111 /$ CC111 & P16 (5) \\
\hline & & & $244 /$ CC244 & P7 (2) \\
\hline & & & $244 /$ CC244 & P15 (1) \\
\hline & & & $253 /$ CC253 & P4 (1) \\
\hline & & & $277 /$ CC277 & P6 (3) \\
\hline & & & 773 & P8 (2) \\
\hline \multirow{6}{*}{ bla $a_{\mathrm{VIM}-4} \mid$ arr-7|aacA4|bla } & \multirow{6}{*}{ FN397623; [19] } & \multirow{6}{*}{31} & $235 /$ CC235 & P2 (16) \\
\hline & & & 235/ CC235 & P17 (3) \\
\hline & & & $235 /$ CC235 & P18 (3) \\
\hline & & & $235 /$ CC235 & P19 (3) \\
\hline & & & $235 /$ CC235 & P20 (4) \\
\hline & & & $235 /$ CC235 & P10 (2) \\
\hline$a a c A 29 a\left|b a_{\mathrm{VIM}-17}\right| a a c A 29 b$ & EU118148; [20] & 1 & $1457 /$ CC235 & P3 (1) \\
\hline$b^{\prime} a_{\text {OXA-10 }}|a a c A 4| b a_{\mathrm{VIM}-2} \mid s m r-2$ & KC527014; this study & 2 & 395/ CC395 & P12 (2) \\
\hline bla $a_{\mathrm{VIM}-2}|\operatorname{aacA7}| d f r B$ & KC527015; this study & 1 & $235 /$ CC235 & P13 (1) \\
\hline
\end{tabular}

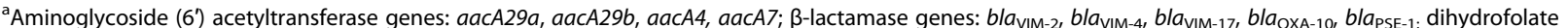
reductase type B (trimethoprim resistance) gene: dfrB; ADP-ribosyl transferase (rifampicin resistance) gene: arr-7; small multidrug resistance protein gene: smr-2.

context and molecular types of VIM-producing $P$. aeruginosa was assessed. Most (46\%) of the VIM producers belonged to the internationally-distributed clusters of ST111 and ST235, which carried previously-reported gene cassettes coding for the VIM-2 and VIM-4 enzymes, respectively. Novel VIM-2-carrying integron structures were identified among sporadic isolates. The VIM-17 carrying integron was found in an isolate of a novel ST (ST1457). The findings of this study underscore the high prevalence of VIM producers among carbapenem-resistant $P$. aeruginosa in Thessaly, as well as their ongoing evolution.

\section{$\langle$ intII}
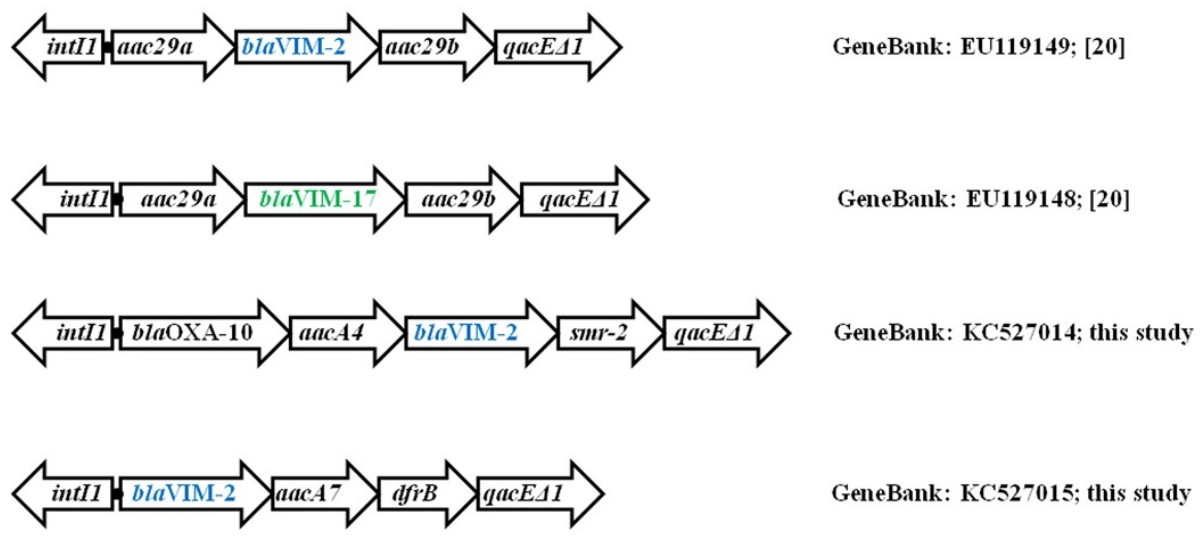

GeneBank: KC527015; this study

Figure 2 Schematic representation (not to scale) of class I integrons of VIM-carrying P. aeruginosa isolates. The GenBank accession numbers of the sequences are also indicated. 


\section{Abbreviations}

CC: Clonal complex; ESBL: Extended-spectrum $\beta$-lactamase; GES: Guiana extended-spectrum $\beta$-lactamase; IBC: Integron-associated class A $\beta$-lactamase; ICU: Intensive care unit; IMP: Imipenemase; KPC: Klebsiella pneumoniae carbapenemase; MBL: Metallo- $\beta$-lactamase; MDR: Multidrug-resistant; MIC: Minimum inhibitory concentration; MLST: Multilocus sequence typing; PCR: Polymerase chain reaction; ST: Sequence type; UHL: University Hospital of Larissa; VIM: Verona integron-encoded metallo- $\beta$-lactamase.

\section{Competing interests}

The authors have no conflicts of interests to declare.

\section{Authors' contributions}

$E P, A M$, and $V M$ conceived and designed the study. $A L$ and $A M$ performed the genetic analysis and molecular typing of isolates. AL and AT collected and reviewed the clinical data. AM was conducted the study, managed the data, and wrote the first draft of the paper. EP and VM interpreted the data. Other co-authors participated in data analysis, data interpretation, and contributed to the final draft. All authors read and approved the final manuscript.

\section{Acknowledgements}

We thank Dr Elli Pinnock for importing isolates and novel profiles at the P. aeruginosa MLST website (http://pubmlst.org/paeruginosa/).

\section{Author details}

${ }^{1}$ Department of Microbiology, University Hospital of Larissa, Larissa, Greece. ${ }^{2}$ Faculty of Biology, Microbiology Group, University of Athens, Athens, Greece. ${ }^{3}$ Department of Urology, University Hospital of Larissa, Larissa, Greece. ${ }^{4}$ Laboratory of Bacteriology, Hellenic Pasteur Institute, Athens, Greece. ${ }^{5}$ Department of Microbiology, Medical School, University of Thessaly, Biopolis, Larissa, Greece.

Received: 18 June 2013 Accepted: 20 October 2013

Published: 29 October 2013

\section{References}

1. Tomás M, Doumith M, Warner M, Turton JF, Beceiro A, Bou G, Livermore DM, Woodford N: Efflux pumps, OprD porin, AmpC b -lactamase, and multiresistance in Pseudomonas aeruginosa isolates from cystic fibrosis patients. Antimicrob Agents Chemother 2010, 54:2219-2224.

2. Weldhagen GF, Poirel L, Nordmann P: Ambler class A extended-spectrum $\beta$-lactamases in Pseudomonas aeruginosa: novel developments and clinical impact. Antimicrob Agents Chemother 2003, 47:2385-2392.

3. Queenan AM, Bush K: Carbapenamases: the versatile $\beta$-lactamases. Clin Microbiol Rev 2007, 20:440-458.

4. Sacha P, Wieczorek P, Hauschild T, Zórawski M, Olszańska D, Tryniszewska E: Metallo-beta-lactamases of pseudomonas aeruginosa-a novel mechanism resistance to beta-lactam antibiotics. Folia Histochem Cytobiol 2008, 46:137-142.

5. Tzouvelekis LS, Markogiannakis A, Psichogiou M, Tassios PT, Daikos GL: Carbapenemases in Klebsiella pneumoniae and other Enterobacteriaceae: an evolving crisis of global dimensions. Clin Microbiol Rev 2012, 25:682-707.

6. Lolans K, Queenan AM, Bush K, Sahud A, Quinn JP: First nosocomial outbreak of Pseudomonas aeruginosa producing-integron borne metallo$\beta$-lactamase (VIM-2) in the United States. Antimicrob Agents Chemother 2005, 49:3538-3540.

7. Rossolini GM, Luzzaro F, Migliavacca R, Mugnaioli C, Pini B, Luca F, Perilli M, Pollini S, Spalla M, Amicosante G, Toniolo A, Pagani L: First countrywide survey of acquired metallo- $\beta$-lactamases in gram-negative pathogens in Italy. Antimicrob Agents Chemother 2008, 52:4023-4029.

8. Patzer JA, Walsh TR, Weeks J, Dzierżanowska D, Toleman MA: Emergence and persistence of integron structures harbouring VIM genes in the children's memorial health institute, Warsaw, Poland, 1998-2006. J Antimicrob Chemother 2009, 63:269-273.

9. Lee K, Lim JB, Yum JH, Yong D, Chong Y, Kim JM, Livermore DM: bla viM-2 cassette-containing novel integrons in metallo- $\beta$-lactamase-producing Pseudomonas aeruginosa and Pseudomonas putida isolates disseminated in a Korean hospital. Antimicrob Agents Chemother 2002, 46:1053-1058.

10. Zhao $\mathrm{WH}, \mathrm{Chen} \mathrm{G}$, Ito $\mathrm{R}$, Hu ZQ: Revelance of resistance levels to carbapenems and integron-borne bla ${ }_{\text {IMP-1, }}$, $l a_{\text {IMP-7, }}$, bla ${ }_{\text {IMP-10 }}$ and bla VIM-2 in clinical isolates of Pseudomonas aeruginosa. J Med Microbio/ 2009 58:1080-1085.
11. Viedma E, Juan C, Villa J, Barrado L, Orellana MA, Sanz F, Otero JR, Oliver A, Chaves F: VIM-2-producing multidrug-resistant Pseudomonas aeruginosa clone ST175, Spain. Emerg Infect Dis 2012, 18:1235-1241.

12. Woodford N, Turton JF, Livermore DM: Multiresistant gram-negative bacteria: the role of high-risk clones in the dissemination of antibiotic resistance. FEMS Microbiol Rev 2011, 35:736-755.

13. Giske CG, Libish B, Colinon C, Scoulica E, Pagani L, Füzi M, Kronvall G, Rossolini GM: Establishing clonal relationships between VIM-1-like metallo- $\beta$-lactamase-producing Pseudomonas aeruginosa strains from four European countries by multilocus sequence typing. J Clin Microbiol 2006, 44:4309-4315.

14. Curran B, Jonas D, Grundmann H, Pitt T, Dowson CG: Development of multilocus sequence typing scheme for the opportunistic pathogen Pseudomonas aeruginosa. Clin Microbiol 2004, 42:5644-5649.

15. Clinical and Laboratory Standards Institute: Performance standards for antimicrobial susceptibility testing: twenty-first informational supplement, M100S21. Wayne, PA, USA: Clinical and Laboratory Standards Institute (CLSI); 2011.

16. Ellington MJ, Kistler J, Livermore DM, Woodford N: Multiplex PCR for rapid detection of genes encoding acquired metallo- $\beta$-lactamases. J Antimicrob Chemother 2007, 59:321-322.

17. Marques ASA, Marchaison A, Gardan L, Samson R: BOX-PCR-based identification of bacterial species belonging to Pseudomonas syringae-P. viridiflava group. Genet Mol Biol 2008, 31:106-115.

18. Feil E, Li BC, Aanensen DM, Hanage WP, Spratt BG: eBURST: inferring patterns of evolutionary descent among clusters of related bacterial genotypes from multilocus sequence typing data. J Bacteriol 2004, 186:1518-1530.

19. Samuelsen $\varnothing$, Toleman MA, Sundsfjord A, Rydberg J, Leegaard TM, Walder M, Lia A, Ranheim TE, Rajendra Y, Hermansen NO, Walsh TR, Giske CG: Molecular epidemiology of metallo-ß-lactamase-producing Pseudomonas aeruginosa isolates from Norway and Sweden shows import of international clones and local clonal expansion. Antimicrob Agents Chemother 2010, 54:346-352.

20. Siarkou VI, Vitti D, Protonotariou E, Ikonomidis A, Sofianou D: Molecular epidemiology of outbreak-related Pseudomonas aeruginosa strains carrying the novel variant bla $\mathrm{VIM}_{-17}$ metallo- $\beta$-lactamase gene. Antimicrob Agents Chemother 2009, 53:1325-1330.

21. Koutsogiannou M, Drougka E, Liakopoulos A, Jelastopulu E, Petinaki E, Anastassiou ED, Spiliopoulou I, Christofidou M: Spread of multidrug resistant Pseudomonas aeruginosa clones in a university hospital. J Clin Microbiol 2013, 51:665-668.

22. Hentschke M, Goritzkaa V, Camposa CB, Merkelb P, Ilchmannc C, Lellekd H, Scherpea S, Aepfelbachera M, Rohdea H: Emergence of carbapenemases in gram-negative bacteria in Hamburg, Germany. Diagn Microbiol Infect Dis 2011, 71:312-315.

23. Larche J, Pouillot F, Essoh C, Libisch B, Straut M, Lee JC, Soler C, Lamarca R, Gleize E, Gabard J, Vergnaud G, Pourcel C: Rapid identification of international multidrug-resistant Pseudomonas aeruginosa clones by multiple-locus variable number of tandem repeats analysis and investigation of their susceptibility to lytic bacteriophages. Antimicrob Agents Chemother 2012, 56:6175-6180.

24. Cabot G, Ocampo-Sosa AA, Dominguez MA, Gago JF, Juan C, Tubau F, Rodriguez C, Moya B, Pena C, Martinez-Martinez L, Oliver A: Genetic markers of widespread extensively drug-resistant Pseudomonas aeruginosa high-risk clones. Antimicrob Agents Chemother 2012, 56:6349-6357.

25. Vatcheva-Dobrevska R, Mulet X, Ivanov I, Zamorano L, Dobreva E, Velinov T, Kantardjiev T, Oliver A: Molecular epidemiology and multidrug resistance mechanisms of Pseudomonas aeruginosa isolates from Bulgarian hospitals. Microb Drug Resist 2013, 19(5):335-361. Epub ahead of print.

26. Pollini S, Antonelli A, Venturelli C, Maradei S, Veggetti A, Bracco S, Rumpianesi F, Luzzaro F, Rossolini GM: Acquisition of plasmid-borne blalMP-19 gene by a VIM-1-positive Pseudomonas aeruginosa of the sequence type 235 epidemic lineage. J Antimicrob Chemother 2013, 68:722-724

27. Park YS, Lee H, Chin BS, Han SH, Hong SG, Hong SK, Kim HY, Uhg Y, Shin HB, Choo EJ, Han S-H, Song W, Jeong SH, Lee K, Kim JM: Acquisition of extensive drug-resistant Pseudomonas aeruginosa among hospitalized patients: risk factors and resistance mechanisms to carbapenems. J Hosp Infect 2011, 79:54-58

28. Tsakris A, Pournaras S, Woodford N, Palepou MF, Babini GS, Douboyas J, Livermore DM: Outbreak of infections caused by Pseudomonas aeruginosa producing VIM-1 carbapenemase in Greece. J Clin Microbiol 2000, 38:1290-1292. 
29. Mavroidi A, Tsakris A, Tzelepi E, Pournaras S, Loukova V, Tzouvelekis LS: Carbapenem-hydrolysing VIM-2 metallo- beta-lactamase in Pseudomonas aeruginosa from Greece. J Antimicrob Chemother 2000, 46:1041-1042.

30. Pournaras S, Maniati M, Petinaki E, Tzouvelekis LS, Tsakris A, Legakis NJ, Maniatis AN: Hospital outbreak of multiple clones of Pseudomonas aeruginosa carrying the unrelated metallo- $\beta$-lactamase gene variants bla VIM-2 and bla vIM-4. J Antimicrob Chemother 2003, 51:1409-1414.

doi:10.1186/1471-2334-13-505

Cite this article as: Liakopoulos et al:: Carbapenemase-producing

Pseudomonas aeruginosa from central Greece: molecular epidemiology

and genetic analysis of class I integrons. BMC Infectious Diseases 2013 13:505.

\section{Submit your next manuscript to BioMed Central and take full advantage of:}

- Convenient online submission

- Thorough peer review

- No space constraints or color figure charges

- Immediate publication on acceptance

- Inclusion in PubMed, CAS, Scopus and Google Scholar

- Research which is freely available for redistribution 